\title{
USO DE METILFENIDATO EM CRIANÇAS COM TRANSTORNO DE DÉFICIT DE ATENÇÃO E HIPERATIVIDADE EM UM MUNICÍPIO DO INTERIOR DO PARANÁ, BRASIL
}

\author{
Flavia BARBOSA, Leyde Daiane de PEDER* \& Claudinei Mesquita da SILVA \\ Centro Universitário da Fundação Assis Gurgacz, Cascavel, PR, Brasil. \\ *Autor para correspondência: leydepeder@yahoo.com.br \\ http://dx.doi.org/10.18571/acbm.108
}

\section{RESUMO}

O presente estudo teve por objetivo analisar o uso do medicamento Metilfenidato em crianças diagnosticadas com Transtorno do Déficit de Atenção e Hiperatividade (TDAH), residentes no município de Nova Aurora, Paraná, Brasil. Para tanto, 26 crianças com a patologia foram selecionadas aleatoriamente, e seus dados foram obtidos por questionário pré-elaborado, aplicado aos pais ou responsáveis e aos professores. A maioria $(17 / 65,4 \%)$ foi diagnosticada na faixa etária entre 6-8 anos e pertencia ao sexo masculino $(22 / 84,6 \%)$. Os principais sinais e sintomas relatados foram: agitação $(23 / 88,5 \%)$ e diminuição da concentração (12/46,2\%). Em 16 $(64,0 \%)$ crianças, verificou o aumento da atenção e diminuição da agitação com o uso do Metilfenidato, no entanto, somente $65,4 \%$ dos pais ou responsáveis relataram conhecimento do tratamento. Verificou-se que $10(40,0 \%)$ e $8(32,0 \%)$ das crianças apresentaram perda de apetite e dores de cabeça/episódios de ansiedade como principais efeitos colaterais do medicamento, respectivamente e $8(32,0 \%)$ crianças não apresentaram nenhum efeito colateral. Assim, o uso de Metilfenidato em crianças na região estudada, melhorou a relação interpessoal, aumentou a concentração e diminuiu a agressividade, demonstrando a importância do uso do medicamento em crianças com TDAH. Contudo, efeitos colaterais ainda são relatados com o uso da terapia.

Palavras-chave: Transtorno do Déficit de Atenção com Hiperatividade; Metilfenidato; Criança.

\begin{abstract}
This study aimed to analyze the use of Methylphenidate drug in children diagnosed with Attention Deficit Hyperactivity Disorder (ADHD), residents in Nova Aurora city, Paraná State, Brazil. Therefore, 26 children with the disease were randomly selected, and data were obtained by a pre-prepared questionnaire administered to parents or guardians and teachers. Most (17/65.4\%) were diagnosed in the age group between 6-8 years old and were male (22/84.6\%). The main signs and symptoms reported were: agitation (23/88.5\%) and decreased concentration (12/46.2\%). In 16 $(64.0 \%)$ children, found increased attention and reduced agitation with the use of methylphenidate, however, only $65.4 \%$ of parents or guardians reported knowledge of treatment. It was found that $10(40.0 \%)$ and $8(32.0 \%)$ children had loss of appetite and headaches/episodes of anxiety and major side effects of the drug, respectively, and 8 (32.0\%) children showed no side effects. Thus, the use of methylphenidate in children in the study area, improved interpersonal relationships, increased concentration and decreased aggression, demonstrating the importance of the drug in children with ADHD. However, side effects are still reported with the use of the therapy.
\end{abstract}

Keywords: Attention Deficit Disorder with Hyperactivity; Methilphenydate; Child. 


\section{Introdução}

O Transtorno de Déficit de Atenção e Hiperatividade é um problema neurobiológico, de causas genéticas, que frequentemente acompanha o indivíduo por toda sua vida (SANTOS \& VASCONCELOS, 2010). O TDAH acomete principalmente crianças, sendo caracterizado por distração e dificuldade de concentração, além de hiperatividade e impulsividade (PEREIRA, 2010). A problemática do TDAH vai além dos sintomas relatados, mas a presença dos mesmos, leva a consequências negativas, interferindo no psiquismo, memória, relações familiares, sociais, e posteriormente, na vida profissional do acometido (NETO, 2009).

As estimativas de prevalência da doença no Brasil variam de $0,9 \%$ a 26,8\%. O tratamento é complexo e inclui intervenções sociais, psicológicas, comportamentais e farmacológicas (SAÚDE, 2014), sendo que o fármaco mais frequentemente utilizado é o Metilfenidato (Cloridrato de Metilfenidato). As resoluções federais 344/1998 e 22/2001 incluem o Metilfenidato na Lista A3 (substâncias psicotrópicas) devido ao elevado risco de dependência química (CARLINI et al., 2003) e dos efeitos colaterais em curto prazo, como redução do apetite, insônia, dor abdominal e cefaleia e, em longo prazo, como efeitos cardiovasculares e possível redução da estatura (KLEINSCHWARTZ, 2002).

O Metilfenidato vem sendo utilizado também para fins não médicos nos últimos anos (CRUZ et al., 2011). Em 2011, a maior produção do medicamento foi registrada desde a década de 1990 (NATIONS; NARCOTICS; BOARD, 2010), sendo que o principal produtor foi os Estados Unidos, responsável pela produção de 41 toneladas. Neste mesmo ano, foi comercializado no Brasil um montante aproximado de 413,3 milhões de miligramas (ANVISA et al., 2010). O aumento do consumo do medicamento, inclusive não prescrito, e seu alto potencial para abuso e dependência, evidenciam a necessidade de se fornecer informações seguras e promover o uso racional e cauteloso da droga (SAÚDE, 2014).

Sabe-se que o Metilfenidato tem efeito benéfico sobre o comportamento e o desempenho escolar de crianças, no entanto, poucos estudos abordam os efeitos colaterais do fármaco e os sintomas do TDAH em crianças hiperativas. Assim, o objetivo do presente estudo foi relatar os sintomas das crianças portadoras TDHA, antes e durante o tratamento com o Metilfenidato, bem como, descrever as características epidemiológicas das mesmas, por meio de um estudo descritivo e exploratório em residentes de um município do oeste do estado do Paraná, Brasil.

\section{Materiais e Métodos}

Realizou-se um estudo de corte transversal, cuja amostra estudada foi constituída aleatoriamente por crianças portadoras de TDAH. Foram aplicados questionários específicos préelaborados aos pais ou responsáveis e professores de três escolas públicas e da Associação de Pais e Amigos dos Excepcionas (APAE) frequentados pelas crianças, no período compreendido entre Fevereiro a Agosto de 2015, no município de Nova Aurora - PR. Este se localiza na região Oeste do Paraná, Brasil, possui uma área territorial de $474.011 \mathrm{~km}^{2}$, população de 11.659 habitantes e índice de desenvolvimento de 0,733, conforme estimativa do Instituto Brasileiro de Geografia e Estatística (IBGE), publicada em 2014 (IBGE, 2014).

A coleta de dados ocorreu em dois momentos. No primeiro, foi realizada entrevista com os pais ou responsáveis, que forneceram informações relativas às crianças. No segundo, professores responderam aos questionários específicos a respeito do medicamento e da doença. As variáveis de interesse pesquisadas foram sexo, sinais e sintomas relatados, idade do diagnóstico, prescrição do Metilfenidato, conhecimento a respeito das ações do medicamento, mudança no comportamento ou na dose durante o tratamento. O presente trabalho foi aprovado pelo Comitê de Ética em Pesquisa em Seres Humanos do Centro Universitário da Fundação Assis Gurgacz sob parecer 912.421 de 27/11/2014. 


\section{Resultados}

Todas as crianças portadoras de TDAH que participaram do estudo eram pacientes da Unidade Básica de Saúde (UBS) do município de Nova Aurora, Paraná, Brasil. Além delas, participaram 26 pais ou responsáveis e 28 professores que trabalham ou trabalharam com as mesmas. A maioria das crianças pertencia ao sexo masculino (22,84,6\%). 17 (65,4\%) portadores foram diagnosticados entre 6 a 8 anos de idade o que indica o início da vida escolar da criança como o principal momento para o diagnóstico da TDAH. Do total, apenas $1(3,85 \%)$ paciente permaneceu sem tratamento e em $25(96,15 \%)$ foi introduzida medicação logo após o diagnóstico, sendo que $13(50,0 \%)$, utilizaram apenas Metilfenidato, enquanto que 1 (3,8\%), $14(53,8 \%), 5$ $(19,2 \%)$ e $6(23,1 \%)$ receberam doses de $5 \mathrm{mg}, 10 \mathrm{mg}, 18 \mathrm{mg}$ e $20 \mathrm{mg}$, respectivamente, associados a outros medicamentos (Tabela 1).

Tabela 1. Idade do diagnóstico, sexo e medicamentos prescritos para crianças com TDAH, Nova Aurora, 2015.

\begin{tabular}{cc} 
Variáveis & Frequência (n/\%) \\
\hline Idade do diagnóstico (anos) & $1 / 3,8$ \\
$0-2$ & $4 / 15,4$ \\
$3-5$ & $17 / 65,4$ \\
$6-8$ & $4 / 15,4$ \\
$\geq 9$ & \\
Sexo & $22 / 84,6$ \\
Masculino & $4 / 15,4$ \\
Feminino & \\
Prescrição de medicamentos & $25 / 96,1$ \\
Sim & $1 / 3,85$ \\
Não & \\
Medicamento prescrito & $13 / 50,0$ \\
Metilfenidato & $3 / 11,54$ \\
Outro & $9 / 34,62$ \\
Outro + Metilfenidato & $1 / 3,84$ \\
Nenhum &
\end{tabular}

n, número de crianças. 
Em relação ao diagnóstico da patologia, $17(65,4 \%)$ crianças foram diagnosticadas por neuropediatras, e dessas, 9 (52,9\%) obtiveram o diagnóstico definitivo de TDAH na primeira consulta. Quanto ao comportamento, todas as crianças apresentaram dois ou mais sinais/sintomas sugestivos de TDAH, sendo que, os mais relatados foram agitação, $(23 / 88,5 \%)$ e diminuição da concentração (12/46,2\%) (Tabela 2).

Tabela 2. Sinais e sintomas relatados pelos pais ou responsáveis pelas crianças portadoras de TDAH, Nova Aurora, 2015.

\begin{tabular}{cc}
\hline Sinais/sintomas relatados & Frequência (n/\%) \\
\hline Ansiedade & $1 / 3,8$ \\
Medo & $1 / 3,8$ \\
Indisciplinado & $1 / 3,8$ \\
Diminuição da concentração & $12 / 46,2$ \\
Agressivo & $3 / 11,5$ \\
Irritado & $3 / 11,5$ \\
Agitado & $23 / 88,5$ \\
Choro demasiado & $1 / 3,8$ \\
Falava demais & $1 / 3,8$ \\
Disperso & $5 / 19,2$ \\
Distraído & $2 / 7,7$ \\
Dificuldade de aprender & $2 / 7,7$ \\
Vive no mundo da imaginação & $1 / 3,8$ \\
Nervoso & $1 / 3,8$ \\
Não prestava atenção/Desatento & $4 / 15,4$ \\
Inquieto & $3 / 11,5$ \\
Hiperativo & $1 / 3,8$ \\
Impaciente & $1 / 3,8$ \\
Ausência de medo & $2 / 7,7$
\end{tabular}

n, número de crianças. 
Do total de pais ou responsáveis que participaram da pesquisa, apenas $9(36,0 \%)$ conheciam o medicamento antes da prescrição. Quando questionados sobre a utilidade farmacológica, 17 $(68,0 \%)$ responderam que o mesmo é utilizado para TDAH. Em relação ao comportamento dos pacientes após administração dos medicamentos, todos os responsáveis (25/100\%) relataram mudanças significativas no desenvolvimento escolar e no comportamento em suas atividades diárias (Tabela 3).

Tabela 3. Informações sobre o uso do Metilfenidato e comportamento das crianças durante o tratamento, Nova Aurora, 2015.

\begin{tabular}{|c|c|}
\hline Variáveis & Frequência $(n / \%)$ \\
\hline \multicolumn{2}{|l|}{ Conhecimento sobre Metilfenidato } \\
\hline Sim & $9 / 36,0$ \\
\hline Não & $17 / 64,0$ \\
\hline \multicolumn{2}{|l|}{ Para que serve? } \\
\hline Concentração & $13 / 52,0$ \\
\hline Aprendizado & $1 / 4,0$ \\
\hline Acalmar & $2 / 8,0$ \\
\hline Hiperatividade & $5 / 20,0$ \\
\hline Memória & $1 / 4,0$ \\
\hline Não sabe responder & $1 / 4,0$ \\
\hline Acalmar e Hiperatividade & $3 / 12,0$ \\
\hline \multicolumn{2}{|l|}{ Para que seu filho faz uso? } \\
\hline Melhorar concentração e atenção & $1 / 4,0$ \\
\hline Hiperatividade e Impulsividade & $1 / 4,0$ \\
\hline Déficit de atenção & $3 / 12,0$ \\
\hline Hiperatividade & $3 / 12,0$ \\
\hline TDAH & $17 / 68,0$ \\
\hline Concentração e Hiperatividade & $1 / 4,0$ \\
\hline Comportamento após tratamento & $25 / 100$ \\
\hline Tranquilo e vigilante ou ligado & $16 / 64,0$ \\
\hline Tranquilo e dedicado & $6 / 24,0$ \\
\hline Compenetrado & $3 / 12,0$ \\
\hline Pacífico & $1 / 4,0$ \\
\hline
\end{tabular}

n, número de crianças. 
Em relação às alterações nas doses do Metilfenidato durante o tratamento, $12(48,0 \%)$ pacientes necessitaram de ajustes na posologia, algumas vezes associadas ao efeito não desejável do medicamento, ou até mesmo pelo fato da dose ser pequena pela idade da criança. Quando questionados sobre os efeitos colaterais causados pelo medicamento, $25(100,0 \%)$ pais ou responsáveis relataram pelo menos um efeito colateral, $22(88,0 \%)$ relataram que os pacientes faziam uso do Metilfenidato há mais de um ano e quanto ao término do tratamento, apenas 6 $(24,0 \%)$ tem previsão (Tabela 4).

Tabela 4. Mudança na dose, efeitos colaterais e tempo de uso do Metilfenidato dos pacientes portadores de TDAH, Nova Aurora, 2015.

\begin{tabular}{|c|c|}
\hline Variáveis & Frequência $(\mathbf{n} / \%)$ \\
\hline \multicolumn{2}{|l|}{ Houve mudança na dose? } \\
\hline Não & $13 / 52,0$ \\
\hline Sim & $12 / 48,0$ \\
\hline \multicolumn{2}{|l|}{ Mudança na dose há quanto tempo? } \\
\hline$<1$ mês & $3 / 25,0$ \\
\hline 1 mês a 1 ano & $1 / 8,3$ \\
\hline$>1$ ano & $8 / 66,7$ \\
\hline \multicolumn{2}{|l|}{ Motivo da mudança de dose } \\
\hline Ausência de efeito após algum tempo & $10 / 83,3$ \\
\hline Idade & $2 / 16,7$ \\
\hline \multicolumn{2}{|l|}{ Efeitos colaterais mais comuns } \\
\hline Perda de apetite & $10 / 40,0$ \\
\hline Dor de cabeça & $8 / 32,0$ \\
\hline Ansiedade & $7 / 28,0$ \\
\hline Irritabilidade & $4 / 16,0$ \\
\hline Insônia & $3 / 12,0$ \\
\hline Taquicardia & $1 / 4,0$ \\
\hline Outros & $2 / 8,0$ \\
\hline Sem efeitos colaterais & $8 / 32,0$ \\
\hline \multicolumn{2}{|l|}{ Tempo de uso do medicamento (Anos) } \\
\hline$<1$ ano & $3 / 12,0$ \\
\hline $1-2$ & $8 / 32,0$ \\
\hline $3-5$ & $8 / 32,0$ \\
\hline $6-8$ & $4 / 16,0$ \\
\hline$\geq 9$ & $2 / 8,0$ \\
\hline \multicolumn{2}{|l|}{ Previsão para o termino do tratamento } \\
\hline Não há & $19 / 76,0 \%$ \\
\hline Sim, com 18 anos & $3 / 12,0 \%$ \\
\hline Sim, após o término dos estudos & $3 / 12,0 \%$ \\
\hline
\end{tabular}

n, número de crianças. 
Em relação aos 28 professores que participaram da pesquisa, $27(96,4 \%)$ tinham conhecimento sobre o TDAH e $24(85,7 \%)$ participaram no diagnóstico das crianças. Quanto ao comportamento relatado em sala de aula antes do diagnóstico, a maioria relatou episódios semelhantes ao relatados pelos pais (Tabela 5).

Tabela 5. Comportamento em sala de aula antes e durante o tratamento com o Metilfenidato, Nova Aurora, 2015.

\begin{tabular}{cc}
\hline Comportamento & Frequência (n/\%) \\
\hline Pré-tratamento & $25 / 96,1$ \\
Inquietação/Agitação & $13 / 50,0$ \\
Falta de concentração & $13 / 50,0$ \\
Agressividade & $5 / 19,2$ \\
Irritação & $5 / 19,2$ \\
Desatenção & $1 / 3,8$ \\
Impaciência & \\
Durante o tratamento & $12 / 42,8$ \\
Calma e atenta & $12 / 42,8$ \\
Calma e concentrada & $4 / 14,2$ \\
Melhora no aprendizado &
\end{tabular}

n, número de crianças.

\section{Discussão}

Uma das principais características em indivíduos com TDAH é a desatenção e/ou hiperatividade-impulsividade, que geralmente comprometem o funcionamento escolar e social, com maior frequência e intensidade do que as observadas em crianças não portadoras da patologia do mesmo sexo e idade (DIAGNÓSTICOS MENTAIS, 2013). Estudo reportou que as principais dificuldades apresentadas por crianças com TDAH foram dificuldade de atenção e manter-se vigilante, sendo mais evidente em situações que requerem atenção por longos períodos de tempo e durante a realização de tarefas repetitivas, como ocorre na escola (HARPIN, 2005). Os mesmos efeitos foram reportados por Frank-Briggs (2011), o qual relatou em seu estudo que 80\% dos indivíduos com diagnóstico de TDAH apresentam sintomas tanto de desatenção quanto de hiperatividade-impulsividade (FRANK-BRIGGS, 2011). Para Faraone et al (2003), o TDAH pode ser diagnosticado em crianças entre 6 a 8 anos de idade, dados que colaboram com os observados no presente estudo (FARAONE et al., 2003).

Estudos relatados por Rodhe et al (2000), demonstraram que crianças e adolescentes com TDAH apresentam melhoras significativas com o uso correto dos medicamentos, inclusive com relação aos sintomas de desatenção, hiperatividade e ou impulsividade (ROHDE \& BARBOSA, 2000). O Metilfenidato é um dos principais fármacos utilizados para tratamento do TDAH, considerado um estimulante do sistema nervoso central (SNC), que age bloqueando a recaptação de catecolaminas e aumentando o nível de produção de neurotransmissores fundamentais para a memória, a atenção e a regulação de humor. Seu principal emprego se faz no âmbito educacional, principalmente em crianças com TDAH, no intuito de diminuir a inquietação motora e aumentar a concentração (SAÚDE, 2014). Os resultados reportados neste estudo colaboram com essas afirmações. Verificou-se que após a administração do Metilfenidato, todos os responsáveis relataram mudanças significativas no desenvolvimento escolar e no comportamento em suas atividades diárias.

Andrade e Sheurer (2004) reportaram melhora dos sintomas gerais com uso do Metilfenidato, verificando-se melhora significante na hiperatividade. Além disso, observou-se que 
todas as crianças que participaram do estudo apresentaram algum tipo de melhora clínica no que se refere à sintomatologia do TDAH (ANDRADE; SCHEUER, 2004). Itaborahy (2009) descreve em seu estudo melhoras clínicas no desempenho das crianças em suas atividades acadêmicas (ITABORAHY, 2009). Estudo realizado por Andrade e Scheurer (2004) utilizando o questionário de Conners para pais e professores, observou efeito significativo do Metilfenidato em relação à hiperatividade, impulsividade e atenção (ANDRADE; SCHEUER, 2004). Estes dados colaboram com os achados no presente estudo.

Pastura e Mattos (2004) relataram a redução de apetite e insônia como os principais efeitos colaterais do Metilfenidato. Além disso, outros sintomas como ansiedade, tristeza, desinteresse e "olhar parado" são sintomas comumente descritos (PASTURA; MATTOS, 2004). Ahmann et al (1993) e Efron et al (1997) observaram que certos sintomas atribuídos ao medicamento como efeitos colaterais, são na verdade, características do TDAH (AHMANN et al., 1993)(EFRON et al., 1997). Santos (2010) relatou em seu estudo como efeitos colaterais redução do apetite, anorexia, insônia, ansiedade, irritabilidade, labilidade emocional, cefaleia, dores abdominais, alterações do humor, tiques e pesadelos (SANTOS; VASCONCELOS, 2010). Segundo Breggin (1999) o Metilfenidato poderia afetar praticamente todos os sistemas do corpo humano, provocando sintomas mentais, neurológicos, cardiovasculares, gastrointestinais e endócrinometabólicos (BREGGIN, 1999). Assim, a retirada brusca desse medicamento pode causar efeito rebote, desencadeando insônia, depressão e exaustão vespertina (BREGGIN, 1999).

Sabec et al (2009) relatam que na maioria dos casos de TDAH, quem percebe os sintomas nas crianças são os professores e não os pais. Isso se deve ao fato de que as crianças passam a maior parte do tempo sendo observadas pelos professores na sala de aula, ao contrário dos pais, que ficam menos tempo com seus filhos, conforme visualizado no presente estudo. Para Vilanova (1994), é na pré-escola ou na fase escolar que as crianças apresentam maior dificuldade em manter o foco em uma determinada atividade, distraindo-se facilmente (VILANOVA, 1994).

\section{Conclusão}

Dentre as crianças avaliadas no presente estudo, a maioria apresentava falta de concentração e agitação antes do diagnóstico, o que foi solucionado parcialmente, verificando-se melhoras clínicas significativas. A maioria foi diagnosticada no início da idade pré-escolar, demonstrando assim, a grande participação dos professores e pouco conhecimento sobre o transtorno por parte dos pais neste processo, recaindo sobre a escola relatar a suspeita da doença.

O uso do Metilfenidato deve ser indicado em casos estritamente necessários devido aos seus potentes efeitos colaterais. É necessária a avaliação concomitante a tratamentos psicológico ou comportamental, o que não foi avaliado neste estudo.

\section{Agradecimentos}

Os autores gostariam de agradecer a Secretaria Municipal de Saúde de Nova Aurora, em especial as farmacêuticas Dr ${ }^{\mathrm{a}}$. Manuela Aparecida Soares Dourado e Dr ${ }^{\mathrm{a}}$. Fernanda Esse Narciso e a Fundação Araucária pela bolsa fornecida para a realização da pesquisa.

\section{Referências}

AHMANN, P. A. et al. Placebo-controlled evaluation of Ritalin side effects. Pediatrics, v. 91, n. 6, p. 1101-1106, 1993.

ANDRADE, E. R. DE; SCHEUER, C. Análise da eficácia do metilfenidato usando a versão abreviada do questionário de Conners em transtorno de déficit de atenção/hiperatividade. 
Arquivos de Neuropsiquiatria, v. 62, n. 1, p. 81-85, 2004.

ANVISA et al. SNGPC- Farmacoepidemiologia. prescrição e consumo de Metilfenidato no Brasil: identificando riscos para o monitoramento e controle sanitário. Statistics, v. 2, n. 2, p. 1-14, 2010.

BREGGIN, P. R. Psychostimulants in the treatment of children diagnosed with ADHD: Risks and mechanism of action. International Journal of Risk and Safety in Medicine, v. 12, n. Netherlands LG-English PT-Journal: Review EM-199900 DD-20070327, p. 3-35, 1999.

CARLINI, E. A. et al. Metilfenidato: influência da notificação de receita A (cor amarela) sobre a prática de prescrição por médicos brasileiros. Rev. psiquiatr. clín. (Säo Paulo), v. 30, n. 1, p. 1120, 2003.

CRUZ, T. C. S. C. et al. Uso não prescrito de metilfenidato entre estudantes de medicina da universidade federal da Bahia. Gaz. méd. Bahia, v. 81, n. 1, p. 3-6, 2011.

DIAGN, M.; MENTAIS, S. D. E. T. Manual Diagnóstico e Estatístico de Transtornos MentaisDSM-5. [s.l: s.n.].

EFRON, D. et al. Side Effects of Methylphenidate and Dexamphetamine in Children With Attention Deficit Hyperactivity Disorder: A Double-blind, Crossover Trial. Pediatrics, v. 100, n. 4, p. 662-666, 1997.

FARAONE, S. V et al. The worldwide prevalence of ADHD: is it an American condition? World psychiatry : official journal of the World Psychiatric Association (WPA), v. 2, n. 2, p. 104-113, 2003.

FRANK-BRIGGS, A. I. Attention deficit hyperactivity disorder (ADHD). Journal of Pediatric Neurology, v. 9, n. 3, p. 291-298, 2011.

HARPIN, V. A. The effect of ADHD on the life of an individual, their family, and community from preschool to adult life. Archives of disease in childhood, v. 90 Suppl 1, p. i2-7, 2005.

INSTITUTO BRASILEIRO DE GEOGRAFIA E ESTATÍSTICA. IBGE | Cidades | Paraná. Disponível em: 〈http://cidades.ibge.gov.br/xtras/uf.php?lang=\&coduf=41\&search=parana> .

ITABORAHY, C. A Ritalina no Brasil: uma década de produção, divulgação e consumo. Universidade do Estado do Rio de Janeiro. Instituto de Medicina Social, 2009.

KLEIN-SCHWARTZ, W. Abuse and toxicity of methylphenidate. Current opinion in pediatrics, v. 14, n. 2, p. 219-223, abr. 2002.

NATIONS, U.; NARCOTICS, I.; BOARD, C. Psychotropic Substances. [s.l: s.n.].

NETO, M. R. L. TDAH ao longo da vida. [s.1.] Artmed Editora, 2009.

PASTURA, G.; MATTOS, P. Efeitos colaterais do metilfenidato. Revista de Psiquiatria Clinica, v. 31, n. 2, p. 100-104, 2004.

PEREIRA, A. e, Bel, ED. Metilfenidato-principal tratamento para o Déficit de Atenção e 
Hiperatividade: características neuroquímicas e seus efeitos em modelos experimentais. Neurobiologia, v. 73, n. 2, p. 127-132, 2010.

ROHDE, L.; BARBOSA, G. Transtorno de déficit de atenção/hiperatividade. Revista Brasileira de Psiquiatria, v. 22, n. Supl II, p. 7-11, 2000.

SABEC, D. K. et al. Acompanhamento de pacientes com Transtorno do Déficit de Atenção e Hiperatividade (TDAH) em Tratamento. p. 223-229, 2009.

SANTOS, L. DE F.; VASCONCELOS, L. A. Transtorno do Déficit de Atenção e Hiperatividade em Crianças: Uma Revisão Interdisciplinar. v. 26, n. 4, p. 717-724, 2010.

SAÚDE, B. B. DE A. DE T. EM. Metilfenidato no tratamento de crianças com transtorno de déficit de atenção e hiperatividade. In: Portal anvisa. [s.l: s.n.]. v. 23p. 1-18.

VILANOVA, L. C. P. Distúrbios da atençäo na infância e na adolescência. In: Psiquiatria da infância e da adolescência. [s.1.] Santos, 1994. p. 237-240. 